\title{
Investigation of Interaction Solutions for Modified Korteweg-de Vries Equation by Consistent Riccati Expansion Method
}

\author{
Jin-Fu Liang $\mathbb{D}^{1}$ and Xun Wang $\mathbb{D}^{2}$ \\ ${ }^{1}$ School of Physics and Electronic Science, Guizhou Normal University, Guiyang 550025, China \\ ${ }^{2}$ Key Laboratory of Speech Acoustics and Content Understanding, Institute of Acoustics, Chinese Academy of Sciences, Beijing 100190, \\ China \\ Correspondence should be addressed to Jin-Fu Liang; liang.shi2007@163.com
}

Received 29 March 2019; Accepted 2 July 2019; Published 18 July 2019

Guest Editor: Shaowu Li

Copyright @ 2019 Jin-Fu Liang and Xun Wang. This is an open access article distributed under the Creative Commons Attribution License, which permits unrestricted use, distribution, and reproduction in any medium, provided the original work is properly cited.

A consistent Riccati expansion (CRE) method is proposed for obtaining interaction solutions to the modified Korteweg-de Vries $(\mathrm{mKdV})$ equation. Using the CRE method, it is shown that interaction solutions such as the soliton-tangent (or soliton-cotangent) wave cannot be constructed for the $\mathrm{mKdV}$ equation. More importantly, exact soliton-cnoidal periodic wave interaction solutions are presented. While soliton-cnoidal interaction solutions were found to degenerate to special resonant soliton solutions for the values of modulus ( $n$ ) closer to one (upper bound of modulus) in the Jacobi elliptic function, a normal kink-shaped soliton was observed for values of $n$ closer to zero (lower bound).

\section{Introduction}

The direct study of exact solutions to nonlinear evolution equations (NLEEs) has received much attention from many mathematicians and physicists due to the fact that new strides in nonlinear science, which were made possible by a substantial increase in computational platforms such as Mathematica, Maple, and MATLAB, have enabled improvements in the performance of complicated and tedious numerical computational methods. Indeed, several powerful methods such as the Tanh-function method [1-3], F-expansion method [4], Jacobian elliptic function method [5], and variational approach $[6,7]$ have been proposed for constructing exact solutions to NLEEs. Despite the successful implementation of such methods, it is still challenging to obtain solutions for interactions among different types of nonlinear excitations such as the soliton-soliton interaction.

Recently, some new soliton structure solutions were obtained for nonlinear systems. Chen et al. studied the vortex solitons in Bose-Einstein condensates with spin-orbit coupling and Gaussian optical lattices, based on the analytical and numerical method [8]. Milan et al. found exact fundamental soliton solutions in the spiraling guiding structures by the modified Petviashvilis iteration method [9]. Cheng et al. investigated the formation and propagation of a multipole soliton in a cold atomic gas with a parity-time symmetric potential using the modified square operator method [10]. Liu et al. obtained the three-soliton solutions for high-order nonlinear Schrodinger equation by Hirotas bilinear method [11].

Specially, Lou [12] proposed a consistent Riccati expansion (CRE) method, which is a more generalized yet simpler method to find interaction solutions for various NLEEs [1317]. The core concept of CRE is the construction of interaction solutions based on the usual Riccati equation method and the consistent equation or the $w$-equation [12]. The CRE method is critical to finding more new solutions to the $w$-equation.

In this study, the CRE method is used to construct several types of interaction solutions for the focusing real modified Korteweg-de Vries (mKdV) equation [18] shown in

$$
u_{t}+\alpha u^{2} u_{x}+\beta u_{x x x}=0 \text {, }
$$

where $\alpha$ and $\beta$ are arbitrary constants. The $\mathrm{mKdV}$ equation plays an important role in describing some physical phenomena, such as optical cycles $[19,20]$, soliton propagation in 
plasma [21] and lattices [22], the Schottky barrier transmission lines [23], and fluid mechanics [24].

To provide better insights into these physical phenomena, finding and analyzing exact solutions to the $\mathrm{mKdV}$ equation is important. Previously, many powerful methods have been proposed for constructing exact solutions to the $\mathrm{mKdV}$ equation. For instance, in 1972, Hirota obtained an exact solution to the $\mathrm{mKdV}$ equation for the case of multiple collisions of solitons with different amplitudes [25]. Subsequently, he also derived the exact envelope soliton solution to the mKdV equation [26]. In 1973, Ablowitz et al. obtained exact solutions to the $\mathrm{mKdV}$ equation by using the inverse scattering technique [27]. In 1988, Akhmediev et al. used the Darboux transformation scheme to obtain second-order periodic solutions to the $\mathrm{mKdV}$ equation [28]. In 2004, Kevrekidis et al. derived some classes of periodic solutions to the mKdV equation by using direct methods [29]. In 2015, Jiao and Lou constructed a new soliton-cnoidal periodic wave interaction solution by using the CRE method [30]. However, they did not investigate how the soliton-cnoidal interaction solutions may be used to derive soliton-soliton or solitonperiodic wave interaction solutions among other types of solutions. Moreover, new interaction solutions to the $\mathrm{mKdV}$ equation involving different types of nonlinear waves must be investigated in depth.

The present article is structured as follows. Section 2 introduces the CRE solvability of the $\mathrm{mKdV}$ equation. Section 3 describes new explicit interaction solutions such as soliton-soliton, multiple resonant soliton, soliton-cosine wave, and soliton-cnoidal wave solutions to the $\mathrm{mKdV}$ equation obtained using the CRE method. Furthermore, it is demonstrated that interaction solutions such as the soliton-tangent wave solution cannot be constructed for the $\mathrm{mKdV}$ equation. The last section presents a summary and discussion.

\section{CRE Solvability of the mKdV Equation}

Consider the following NLEE, shown in (2), with independent variables $X \equiv\left(t, x_{1}, x_{2}, \ldots, x_{m}\right)$ and a dependent variable $u \equiv u(X)$

$$
P\left(u, u_{t}, u_{x_{i}}, u_{x_{i} x_{i}}\right)=0,
$$

where $P$ is a polynomial function of some arguments with the subscripts denoting partial derivatives. We assume that the solution to (2) is the following possible truncated expansion form

$$
u=\sum_{j=0}^{n} u_{j} R^{j}(w)
$$

where $n$ is determined from the leading order analysis of (2). All the expansion coefficient functions $\left(u_{j}\right)$ are determined by substituting (3) into (2) and then vanishing all the coefficients for a given power of $R(w)$. Further, $u_{j}$ and $w$ are functions of $(x, y, t)$ and $R(w)$ satisfies the following simple Riccati equation shown below:

$$
\begin{gathered}
R_{w}=\sigma+R^{2}, \\
R \equiv R(w),
\end{gathered}
$$

which includes the following five special solutions [31]. For $\sigma<0$,

$$
\begin{aligned}
& R=-\sqrt{-\sigma} \tanh (\sqrt{-\sigma} \omega), \\
& R=-\sqrt{-\sigma} \operatorname{coth}(\sqrt{-\sigma} \omega) .
\end{aligned}
$$

For $\sigma>0$,

$$
\begin{aligned}
& R=\sqrt{\sigma} \tan (\sqrt{\sigma} w), \\
& R=-\sqrt{\sigma} \cot (\sqrt{\sigma} w) .
\end{aligned}
$$

For $\sigma=0$,

$$
R=-\frac{1}{w}
$$

Definition. If the equation for $u_{j}(j=0,1, \ldots, n)$ and $w$, obtained by vanishing all the coefficients of each power in $R(w)$ after the substitution of (3) into (2), is either consistent or not overdetermined, then the expansion in (3) is considered a CRE and the nonlinear system in (2) is said to be CRE solvable [8].

According to the CRE method defined above, one can obtain the following form based on the leading order analysis of the mKdV equation in (1)

$$
u=u_{0}+u_{1} R(w)
$$

where $u_{0}, u_{1}$, and $w$ are functions of $(x, y, t)$ and $R(w)$ satisfies the Riccati equation (see (4) above).

Substituting (8) and (4) into (1) and vanishing all the coefficients of different powers of $R(w)$, one obtains

$$
\begin{aligned}
u_{1} & =-\frac{\sqrt{6 \beta} w_{x}}{\sqrt{-\alpha}}, \\
\text { or } u_{1} & =\frac{\sqrt{6 \beta} w_{x}}{\sqrt{-\alpha}}, \\
u_{0} & =-\frac{\sqrt{3 \beta} w_{x x}}{\sqrt{-2 \alpha} w_{x}} \\
\text { or } u_{0} & =\frac{\sqrt{3 \beta} w_{x x}}{\sqrt{-2 \alpha} w_{x}}, \\
w_{t} & =2 \sigma \beta w_{x}^{3}-\frac{3 \beta w_{x x}^{2}}{2 w_{x}}+\beta w_{x x x} .
\end{aligned}
$$

Based on the definition above, (11) is the consistent equation of the $\mathrm{mKdV}$ equation (or the $\mathrm{mKdV} w$-equation). If $w$ is a solution to the MDWW $w$-equation in (11), the $\mathrm{mKdV}$ equation in (1) is CRE solvable. In this study, we set $\alpha=-6$ and $\beta=1$. Thus, the solutions to the $\mathrm{mKdV}$ equation are expressed as follows.

$$
\begin{aligned}
& u=-\frac{w_{x x}}{2 w_{x}}-w_{x} R(w), \\
& u=\frac{w_{x x}}{2 w_{x}}+w_{x} R(w) .
\end{aligned}
$$




\section{Interaction Solutions to the mKdV Equation}

Upon the determination of solutions to (11) by using (12), the corresponding solutions to the $\mathrm{mKdV}$ equation in (1) can be obtained. In this section, we construct interaction solutions to the $\mathrm{mKdV}$ equation by using different types of trivial solutions to (11).

3.1. Soliton-Soliton Interaction Solutions to the $m K d V$ Equation. To obtain soliton-soliton interaction solutions to the $\mathrm{mKdV}$ equation, we consider the following form in (13) as the trial solution to (11):

$$
\begin{aligned}
& w=k_{1} x+w_{1} t+R(\phi), \\
& \phi=k_{2} x+w_{2} t,
\end{aligned}
$$

where $R(\phi)$ satisfies the following Riccati equation in

$$
R_{\phi}=r+R(\phi)^{2}
$$

where $r$ is an arbitrary constant. This equation has special solutions similar to those in (5a), (5b), (6a), (6b), and (7). By vanishing all the coefficients for each power of $R_{\phi}$ after the substitution of (13) and (14) into the mKdV $w$-equation in (11), one can obtain

$$
\begin{gathered}
\sigma=-\frac{1}{4} \\
r=-1 \\
k_{2}=-\sqrt[3]{\frac{w_{2}}{2 r}}, \\
k_{1}=\frac{k_{2} w_{1}}{w_{2}}, \\
w_{1}=-w_{2} .
\end{gathered}
$$

From (15), it can be seen that both $\sigma$ and $r$ are less than 0 when $w_{1} \neq 0$. Based on (5a), (5b), (6a), and (6b), Eqs. (4) and (14) have only solitary solutions (viz., (5a) and (5b)) but not tangent or cotangent solutions such as the ones described in (6a) and (6b). This shows that interaction solutions such as soliton-tangent (or soliton-cotangent) wave cannot be constructed for the $\mathrm{mKdV}$ equation.

Under condition (15), from (5a), (5b), (12a), and (13), the soliton-soliton interaction solutions of the $\mathrm{mKdV}$ equation are expressed as

$$
\begin{aligned}
u= & \frac{2 k_{2}^{2} \tanh \phi}{k_{1}+2 k_{2}+k_{1} \cosh (2 \phi)}+\frac{1}{2}\left(k_{1}+k_{2} \operatorname{sech}^{2} \phi\right) \\
& \cdot \tanh \left(\frac{1}{2}\left(-k_{1} x-w_{1} t\right)-\tanh \phi\right),
\end{aligned}
$$

$$
\begin{aligned}
u= & \frac{k_{2}^{2} \operatorname{csch}^{2} \phi \operatorname{coth} \phi}{-k_{1}+k_{2} \operatorname{csch}^{2}(\phi)}+\frac{1}{2}\left(k_{1}-k_{2} \operatorname{csch}^{2} \phi\right) \\
& \cdot \tanh \left(\frac{1}{2}\left(-k_{1} x-w_{1} t\right)-\operatorname{coth} \phi\right), \\
u= & \frac{k_{2}^{2} \operatorname{sech}^{2} \phi \tanh \phi}{k_{1}+k_{2} \operatorname{sech}^{2}(\phi)}+\frac{1}{2}\left(k_{1}+k_{2} \operatorname{sech}^{2} \phi\right) \\
& \cdot \operatorname{coth}\left(\frac{1}{2}\left(-k_{1} x-w_{1} t\right)-\tanh \phi\right), \\
u= & \frac{-k_{2}^{2} \operatorname{coth} \phi \operatorname{csch}^{2} \phi}{k_{1}-k_{2} \operatorname{csch}^{2}(\phi)}+\frac{1}{2}\left(k_{1}-k_{2} \operatorname{csch}^{2} \phi\right) \\
& \cdot \operatorname{coth}\left(\frac{1}{2}\left(-k_{1} x-w_{1} t\right)-\operatorname{coth} \phi\right) .
\end{aligned}
$$

3.2. Interaction Solution between and Trigonometric Periodic Wave for the $m K d V$ Equation. To investigate the interaction between a soliton and a periodic wave in the interaction solution to the mKdV equation, we consider a solution of the following form (11):

$$
\begin{aligned}
w= & k_{1} x+w_{1} t \\
& +c_{0} \ln \left(\cos \left(k_{2} x+w_{2} t\right) \exp \left(k_{2} x+w_{2} t\right)\right),
\end{aligned}
$$

where $c_{0}$ is determined constant. Substituting (17) into (11) and then vanishing all coefficients of powers $\tan \left(k_{2} x+w_{2} t\right)$, we can obtain

$$
\begin{aligned}
\sigma & =-\frac{1}{4}, \\
k_{1} & =-\frac{k_{2}}{\sqrt{-2 \sigma}}, \\
c_{0} & =\frac{2\left(2 \sigma k_{1}^{3} k_{2}-k_{1} k_{2}^{3}\right)}{k_{2}^{4}-8 \sigma k_{1}^{2} k_{2}^{2}+4 \sigma^{2} k_{1}^{4}}, \\
w_{1} & =\frac{2\left(2 c_{0} k_{1} k_{2}^{3}+3 c_{0}^{2} k_{2}^{4}-\sigma k_{1}^{4}\right)}{k_{1}}, \\
w_{2} & =4\left(k_{2}^{3}-3 \sigma k_{1}^{2} k_{2}-6 \sigma c_{0} k_{1} k_{2}^{2}\right) .
\end{aligned}
$$

Then, based on (5a), (17), and (12a), one can obtain a soliton-trigonometric periodic wave interaction solution as

$$
\begin{aligned}
u= & k_{2} \frac{\left(6 \sec ^{2}(M)-\sqrt{2}\left(1+2 \tan ^{2}(M)\right)\right)}{6+12 \tan (M)} \\
& +\frac{\tanh \left(\left(3 k_{2}\left(x+k_{2}^{2} t\right)-2 \ln (M \cos (M))\right) / 3 \sqrt{2}\right)}{6+12 \tan (M)},
\end{aligned}
$$

where $M=k_{2} x+2 k_{2}^{3} t$.

3.3. Interaction Solutions between Soliton and Cnoidal Wave for the $m K d V$ Equation. In [26], Jiao and Lou constructed a solution of the following form for (11)

$$
w=k_{1} x+w_{1} t+A E_{\pi}\left[\operatorname{sn}\left(k_{2} x+w_{2} t, \mu_{1}\right), v, \kappa\right],
$$


where $\operatorname{sn}(\zeta, \mu)$ is the usual Jacobi elliptic sine function and $E_{\pi}(\xi, v, \kappa)$ is the third type of incomplete elliptic integral. Jiao and Lou used the following parameters in (21) to obtain a special soliton-cnoidal wave interaction solution to the mKdV equation:

$$
\left\{\mu_{1}, k_{1}, \mathrm{k}_{2}\right\}=\{1.5,2,1\} \text {. }
$$

As seen from (21), Jiao and Lou chose the modulus $\left(\mu_{1}\right)$ of Jacobi elliptic function to be 1.5, which is outside the allowed range $\left(0<\mu_{1}<1\right)[13]$.

In this study, we will further investigate how solitoncnoidal interaction solutions can be used to derive solitonsoliton and soliton-periodic wave interaction solutions among other types of solutions. To this end, we performed all the substitutions and evaluations by using the Mathematica software.

Consider a trial solution of the following form for solving

$$
w=k_{1} x+w_{1} t+W\left(k_{2} x+w_{2} t\right)
$$

where

$$
W\left(k_{2} x+w_{2} t\right)=W(\xi)=W,
$$

satisfies the following elliptic equation:

$$
\begin{aligned}
W_{1 \xi}^{2} & =C_{0}+C_{1} W_{1}+C_{2} W_{1}^{2}+C_{3} W_{1}^{3}+C_{4} W_{1}^{4}, \\
W_{1} & \equiv W_{\xi} .
\end{aligned}
$$

Substituting (22) and (24) into (11), one obtains

$$
\begin{aligned}
& C_{0}=C_{0}, \\
& C_{1}=\frac{3 C_{0} k_{2}^{4}-2 k_{1} w_{1}-4 \sigma k_{1}^{4}}{k_{1} k_{2}^{3}}, \\
& C_{2}=\frac{C_{1} k_{2}^{4}-k_{2} w_{1}-k_{1} w_{2}-8 \sigma k_{2} k_{1}^{3}}{k_{1} k_{2}^{3}}, \\
& C_{3}=\frac{C_{2} k_{2}^{3}-2 w_{2}-24 \sigma k_{2} k_{1}^{2}}{3 k_{1} k_{2}^{2}}, \\
& C_{4}=-4 \sigma .
\end{aligned}
$$

From the analysis of (24), we assume the solution of (24) in the following form

$$
W_{1}=A_{0}+A_{1} \operatorname{sn}\left(m\left(k_{2} x+w_{2} t\right), n\right) .
$$

Substituting (26) into (24) and setting the coefficient of $\left\{\operatorname{sn}\left(m\left(k_{2} x+w_{2} t\right), n\right), \operatorname{cn}\left(m\left(k_{2} x+w_{2} t\right), n\right), \operatorname{dn}\left(m\left(k_{2} x+\right.\right.\right.$ $\left.\left.\left.w_{2} t\right), n\right)\right\}$ equal to zero, one obtains

$$
\begin{aligned}
& A_{0}=A_{0}, \\
& A_{1}=A_{0}, \\
& C_{0}=\frac{-m^{2} A_{0}^{2} A_{1}^{2}+m^{2} A_{1}^{4}+m^{2} n^{2} A_{0}^{4}-m^{2} n^{2} A_{0}^{2} A_{1}^{2}}{A_{1}^{2}}, \\
& C_{1}=\frac{2\left(2 C_{0}+m^{2} A_{0}^{2}-2 m^{2} A_{1}^{2}+m^{2} n^{2} A_{0}^{2}\right)}{A_{0}}, \\
& C_{2}=\frac{-3 C_{1}+4 m^{2} A_{0}+4 m^{2} n^{2} A_{0}}{2 A_{0}}, \\
& C_{3}=\frac{2\left(C_{2}+m^{2}+m^{2} n^{2}\right)}{3 A_{0}}, \\
& C_{4}=\frac{C_{3}}{4 A_{0}} .
\end{aligned}
$$

Based on (25) and (27), one can find a group solution

$$
\begin{aligned}
& k_{2}=\sqrt[3]{-\frac{2 w_{2}}{m^{2}\left(5-n^{2}\right)}}, \\
& k_{1}=\frac{600 \sigma w_{1} w_{2}-\sqrt{360000 \sigma^{2} w_{1}^{2} w_{2}^{2}-4\left(N_{1}\right)\left(N_{2}\right)}}{2\left(N_{2}\right)}, \\
& A_{0}=\frac{-4 m^{2} k_{1} k_{2}^{3}-4 m^{2} n^{2} k_{1} k_{2}^{3}+3 w_{1} k_{2}+w_{2} k_{1}}{4 k_{2}\left(m^{2} k_{2}^{3}+m^{2} n^{2} k_{2}^{3}-w_{2}\right)},
\end{aligned}
$$

$A_{1}$

$$
=-\sqrt{\frac{6\left(m^{2} n^{2} k_{2} k_{1}^{2}+2 m^{2} n^{2} k_{1} k_{2}^{2} A_{0}+m^{2} n^{2} k_{2}^{2} \mathrm{~A}_{0}^{2}\right)}{m^{2} k_{2}^{3}+m^{2} n^{2} k_{2}^{3}+2 w_{2}},}
$$

where $N_{1}=-160 m^{6} k_{2}^{7}+992 m^{6} n^{2} k_{2}^{7}-236 m^{4} w_{2} k_{2}^{4}-$ $616 m^{4} n^{2} w_{2} k_{2}^{4}+20 m^{4} n^{4} w_{2} k_{2}^{4}+168 m^{2} w_{2}^{2} k_{2}+40 m^{2} n^{2} w_{2}^{2} k_{2}+$ $300 \sigma w_{1}^{2} k_{2}$, and $N_{2}=375 \sigma m^{4} k_{2}^{5}-1950 \sigma m^{4} n^{4} k_{2}^{5}+$ $375 \sigma m^{4} n^{4} k_{2}^{5}+600 \sigma m^{2} w_{2} k_{2}^{2}+600 \sigma m^{2} n^{2} w_{2} k_{2}^{2}$. Under the substitution of (26), (22), and (5a) into (12a), under the conditions imposed by (28), one obtains

$$
\begin{aligned}
u= & \frac{-m A_{1} k_{2}^{2} C D}{2\left(k_{1}+k_{2} A_{0}+k_{2} A_{1} S\right)} \\
& +\frac{2\left(k_{1}+k_{2} A_{0}+k_{2} A_{1} S\right)^{2} \sqrt{-\sigma} \tanh (\sqrt{-\sigma} T)}{2\left(k_{1}+k_{2} A_{0}+k_{2} A_{1} S\right)},
\end{aligned}
$$




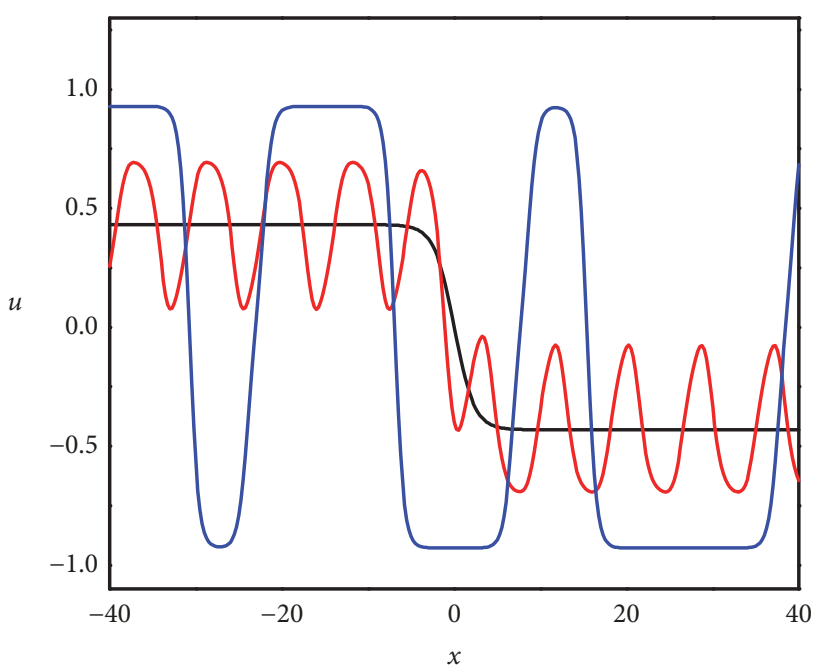

(a)

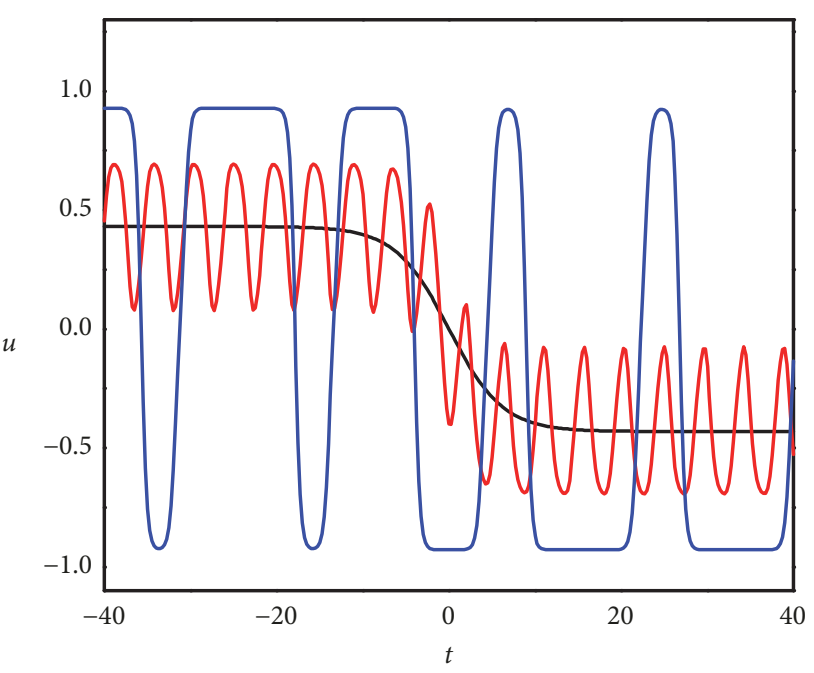

(b)

FIGURE 1: (Color online) The evolution of the soliton-cnoidal wave interaction solution shown in (29) obtained by using the parameters listed in (31). The profiles at $t=0$ and $x=0$ are shown in (a) and (b), respectively. Different color traces represent different values for the modulus $n=0.00001$ (black), $n=0.5$ (red), and $n=0.99999$ (blue).

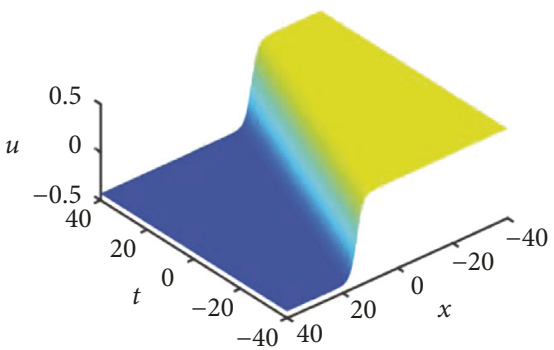

(a)

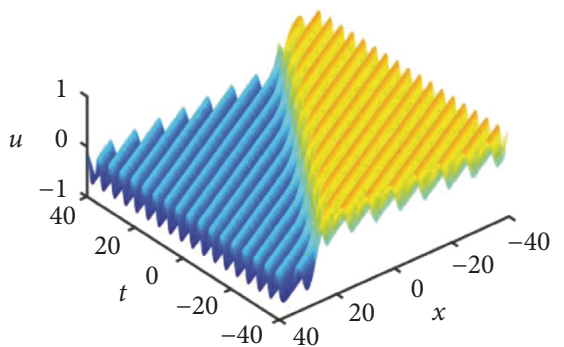

(b)

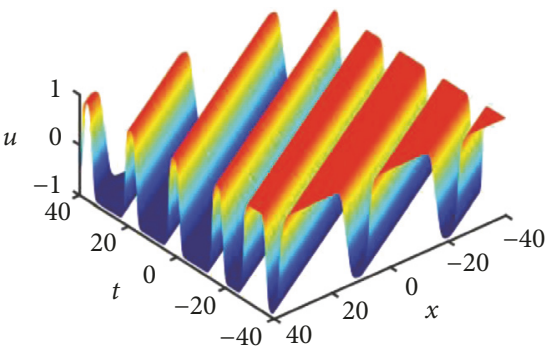

(c)

Figure 2: (Color online) The evolution of the soliton-cnoidal wave interaction solution shown in (29) obtained by using the parameters listed in (31) with different modulus values as a function of both $x$ and $t$. The modulus values for different panels are (a) $n=0.00001$, (b) $n=0.5$, and (c) $n=0.99999$.

where

$$
\begin{aligned}
S= & \operatorname{sn}\left(m\left(k_{2} x+w_{2} t\right), n\right), \\
C= & \operatorname{cn}\left(m\left(k_{2} x+w_{2} t\right), n\right), \\
D= & \operatorname{dn}\left(m\left(k_{2} x+w_{2} t\right), n\right), \\
T= & \left(k_{1}+k_{2} A_{0}\right) x+\left(w_{1}+w_{2} A_{0}\right) t \\
& +\frac{A_{1} \ln (D-\sqrt{n} C)}{m \sqrt{n}} .
\end{aligned}
$$

To investigate how the soliton-cnoidal interaction solutions could be used to derive soliton-soliton interaction or other types of solutions, we illustrate the following two cases corresponding to the soliton-cnoidal wave interaction solution described in (29) by selecting different sets of parameters. For the first case, the parameters are chosen as

$$
\begin{aligned}
\sigma & =-1, \\
w_{1} & =0.5, \\
w_{2} & =0.8, \\
m & =2 .
\end{aligned}
$$

While Figure 1 shows two-dimensional views for interaction solution at $t=0$ and $x=0$. Figure 2 displays threedimensional plots for the evolution of soliton-cnoidal wave interaction solution with different values for the modulus in the Jacobian elliptic function, viz., $n=0.000001,0.5$, and 0.99999 . While $n=0.5$ exhibited a particular periodic-kink soliton wave interaction, the extreme values of $n=0.00001$ (a value close to the lower modulus limit or 0 ) showed a normal kink-shaped soliton and $n=0.9999$ (a value close to the upper modulus limit or 1) displayed an interaction between a periodic wave and another periodic wave.

For the second case, the parameters were altered as shown in (32) by changing the angular frequency $w_{2}$ 


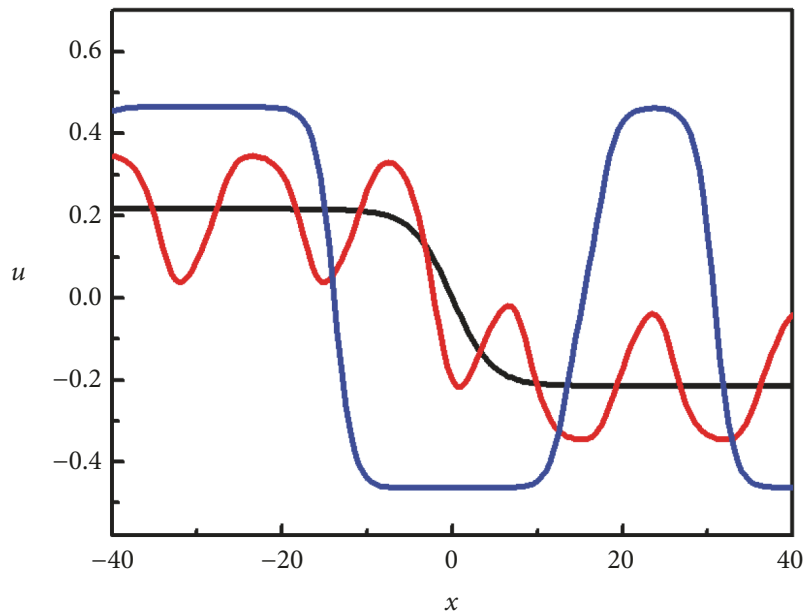

(a)

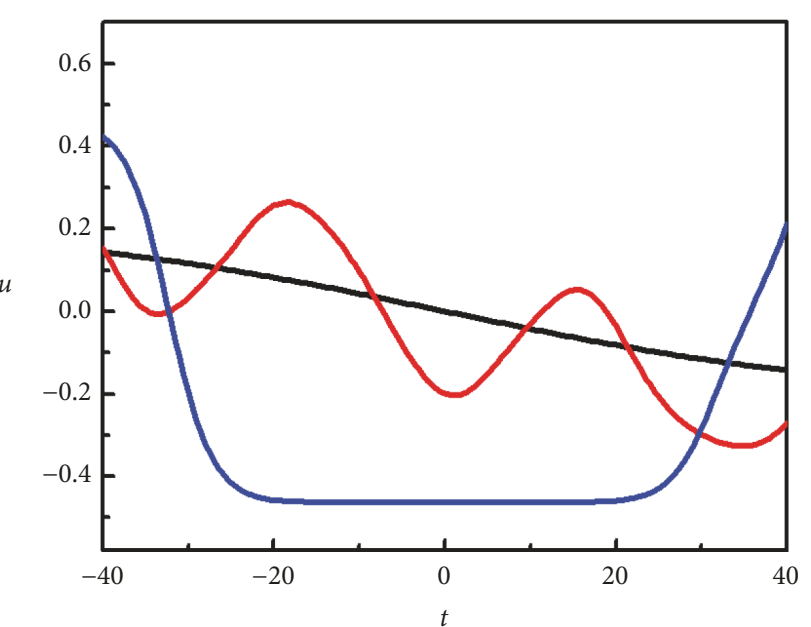

(b)

FIgURE 3: (Color online) The evolution of the soliton-cnoidal wave interaction solution shown in (29) obtained by using the parameters in (32). The profiles at $t=0$ and $x=0$ are shown in (a) and (b), respectively. Different color traces represent different values for the modulus $n$ $=0.00001$ (black), $n=0.5$ (red), and $n=0.99999$ (blue).

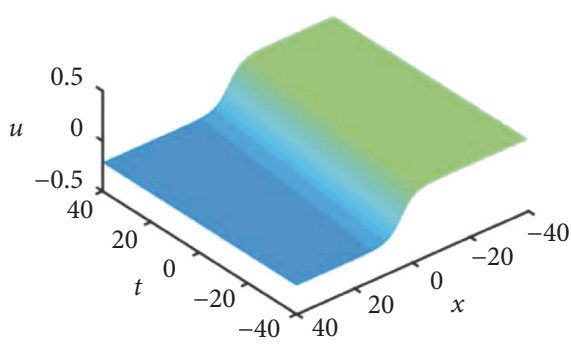

(a)

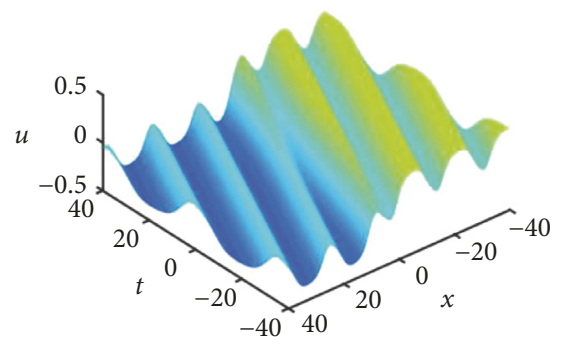

(b)

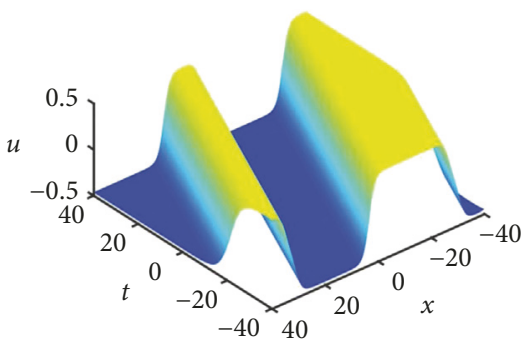

(c)

Figure 4: (Color online) The evolution of the soliton-cnoidal wave interaction solution shown in (29) obtained by using the parameters listed in (32) with different modulus values as a function of both $x$ and $t$. The modulus values for different panels are (a) $n=0.00001,(\mathrm{~b}) n=0.5$, and (c) $n=0.99999$.

$$
\begin{aligned}
\sigma & =-1, \\
w_{1} & =0.5, \\
w_{2} & =0.1, \\
m & =2 .
\end{aligned}
$$

Similar to the first case, we illustrate the structures of the soliton-cnoidal wave interaction solution for different values of $n=0.00001,0.5$, and 0.99999. Clearly, as shown in Figures 3 and 4 , wavenumbers and the amplitudes in the range of $x(-$ $40,40)$ and $t(-40,40)$ are less than that of the first case (cf. Figures 1 and 2 ). While there is still a normal kink soliton in the $x$ - $u$ plot for $n=0.00001$, an incomplete kink soliton is observed in the $t-u$ plot in contrast to the first case shown in Figure 1(b). Building on the above two cases, soliton and soliton-soliton wave interaction solutions are derived from the soliton-cnoidal wave interaction solution by making the limit of the modulus approach either 0 or 1 .

\section{Summary and Discussion}

In this study, we investigated the focusing $\mathrm{mKdV}$ equation by using the CRE method. This nonlinear equation was shown to be CRE solvable and interaction solutions; namely, solitonsoliton, soliton-trigonometric periodic waves, and solitoncnoidal periodic wave for the $\mathrm{mKdV}$ equation were explicitly provided by choosing different trial solutions for the $\mathrm{mKdV}$ $w$-equation shown in (11). In addition, analytical solutions for interactions between soliton and cnoidal wave were provided and their properties were discussed graphically. According to the presented analysis, soliton and soliton-soliton wave interaction solutions can be derived from the soliton-cnoidal wave interaction solution by making the limit of the modulus approach either 0 or 1 (i.e., lower or upper bounds for the modulus in the Jacobi elliptical function).

\section{Data Availability}

No data were used to support this study. 


\section{Conflicts of Interest}

The authors declare that there are no conflicts of interest regarding the publication of this paper.

\section{Acknowledgments}

This work was supported by the National Natural Science Foundation of China (Grant No. 11864007 and No. 11564006), the Doctor Foundation of Guizhou Normal University (2015), and the Science and Technology Planning Project of Guizhou Province (Grant No. [2018]5769).

\section{References}

[1] H. A. Abdusalam, "On an improved complex tanh-function method," International Journal of Nonlinear Sciences and Numerical Simulation, vol. 6, no. 2, pp. 99-106, 2005.

[2] M. A. Abdou and A. A. Soliman, "Modified extended tanhfunction method and its application on nonlinear physical equations," Physics Letters A, vol. 353, no. 6, pp. 487-492, 2006.

[3] D. L. Sekulic, M. V. Sataric, and M. B. Zivanov, "Symbolic computation of some new nonlinear partial differential equations of nanobiosciences using modified extended tanh-function method," Applied Mathematics and Computation, vol. 218, no. 7, pp. 3499-3506, 2011.

[4] W. W. Li, Y. Tian, and Z. Zhang, "F method and its application for finding new exact solutions to the sine-Gordon and sinhGordon equations," Applied Mathematics and Computation, vol. 219, no. 3, pp. 1135-1143, 2012.

[5] A. H. Bhrawy, M. A. Abdelkawy, and A. Biswas, "Cnoidal and snoidal wave solutions to coupled nonlinear wave equations by the extended Jacobi's elliptic function method," Communications in Nonlinear Science and Numerical Simulation, vol. 18, no. 4, pp. 915-925, 2013.

[6] H. Liu, "Variational approach to nonlinear electrochemical system," International Journal of Nonlinear Sciences and Numerical Simulation, vol. 5, no. 1, pp. 95-96, 2004.

[7] J. F. Liang and L. X. Gong, "Complex wave solutions for (2+1)-dimensional modified dispersive water wave system," Communications in Theoretical Physics, vol. 52, no. 1, pp. 17-22, 2009.

[8] S. F. Chen, Q. Guo, S.-L. Xu et al., "Vortex solitons in boseeinstein condensates with spin-orbit coupling and gaussian optical lattices," Applied Mathematics Letters, vol. 92, pp. 15-21, 2019.

[9] S. P. Milan, S. Aleksandra, A. Branislav, and R. Milivoj, "Rotating solitons supported by a spiral waveguide," Physical Review A: Atomic, Molecular and Optical Physics, vol. 98, no. 5, Article ID 063822, 2017.

[10] J. Cheng, S. Xu, M. R. Belić et al., "Multipole solitons in a cold atomic gas with a parity-time symmetric potential," Nonlinear Dynamics, vol. 95, no. 3, pp. 2325-2332, 2019.

[11] X. Liu, H. Triki, Q. Zhou et al., "Generation and control of multiple solitons under the influence of parameters," Nonlinear Dynamics, vol. 95, no. 1, pp. 143-150, 2019.

[12] S. Y. Lou, "Consistent Riccati expansion for integrable systems," Studies in Applied Mathematics, vol. 134, no. 3, pp. 372-402, 2015.

[13] X. Liu, J. Yu, and Z. Lou, "New interaction solutions from residual symmetry reduction and consistent Riccati expansion of the (2+1)-dimensional Boussinesq equation," Nonlinear Dynamics, vol. 92, no. 4, pp. 1469-1479, 2018.

[14] L. Huang, Z. Qiao, and Y. Chen, "Soliton-cnoidal interactional wave solutions for the reduced Maxwell-Bloch equations," Chinese Physics B, vol. 27, no. 2, Article ID 020201, 2018.

[15] J. Chen, H. Wu, and Q. Zhu, "Bäcklund transformation and soliton-cnoidal wave interaction solution for the coupled Klein-Gordon equations," Nonlinear Dynamics, vol. 91, no. 3, pp. 1949-1961, 2018.

[16] Y. Li and H. Hu, "Nonlocal symmetries and interaction solutions of the Benjamin-Ono equation," Applied Mathematics Letters, vol. 75, pp. 18-23, 2018.

[17] J. Liang and X. Wang, "Consistent Riccati expansion for finding interaction solutions of $(2+1)$-dimensional modified dispersive water-wave system," Mathematical Methods in the Applied Sciences, 2019.

[18] S. Liu and S. Liu, Nonlinear Equation in Physics, Peking University Press, Beijing, China, 2000.

[19] H. Leblond and D. Mihalache, "Few-optical-cycle solitons: modified korteweg-de vries sine-gordon equation versus other non-slowly-varying-envelope-approximation models," Physical Review A: Atomic, Molecular and Optical Physics, vol. 79, no. 6, Article ID 063835, 2009.

[20] H. Leblond and D. Mihalache, "Few-optical-cycle dissipative solitons," Journal of Physics A: Mathematical and Theoretical, vol. 43, no. 37, Article ID 375205, 2010.

[21] N. J. Zabusky and M. D. Kruskal, "Interaction of solitons in acollisionless plasma and the recurrence of initial states," Physical Review Letters, vol. 15, no. 6, pp. 240-243, 1965.

[22] H. Ono, "Soliton fission in anharmonic lattices with reflectionless inhomogeneity," Journal of the Physical Society of Japan, vol. 61, no. 12, pp. 4336-4343, 1992.

[23] V. Ziegler, J. Dinkel, C. Setzer, and K. E. Lonngren, "On the propagation of nonlinear solitary waves in a distributed Schottky barrier diode transmission line," Chaos Solitons and Fractals, vol. 12, no. 9, pp. 1719-1728, 2001.

[24] M. A. Helal, "Soliton solution of some nonlinear partial differential equations and its applications in fluid mechanics," Chaos Solitons and Fractals, vol. 13, no. 9, pp. 1917-1929, 2002.

[25] R. Hirota, "Exact solution of the korteweg-de vries equation for multiple Collisions of solitons," Physical Review Letters, vol. 27, no. 18, pp. 1192-1194, 1971.

[26] R. Hirota, "Exact envelope-soliton solutions of a nonlinear wave equation," Journal of Mathematical Physics, vol. 14, pp. 805-809, 1973.

[27] M. J. Ablowitz, D. J. Kaup, A. C. Newell, and H. Segur, "Nonlinear-evolution equations of physical significance," Physical Review Letters, vol. 31, no. 2, pp. 125-127, 1973.

[28] N. Akhmediev, V. I. Korneev, and N. V. Mitskevich, "Nmodulation signals in a single-mode optical fiber with allowance for nonlinearity," Journal of Experimental and Theoretical Physics, vol. 94, pp. 159-170, 1988.

[29] P. G. Kevrekidis, A. Khare, A. Saxena, and G. Herring, "On some classes of $\mathrm{mKdV}$ periodic solutions," Journal of Physics A: Mathematical and General, vol. 37, no. 45, pp. 10959-10965, 2004.

[30] X. Jiao and S. Lou, "CRE method for solving mKdV equation and new interactions between solitons and cnoidal periodic waves," Communications in Theoretical Physics, vol. 63, no. 1, pp. 7-9, 2015. 
[31] S. H. Ma, J. Y. Fang, and C. L. Zheng, "New exact solutions for the (3+1)-dimensional Jimbo-Miwa system," Chaos Solitons and Fractals, vol. 40, no. 3, pp. 1352-1355, 2009. 


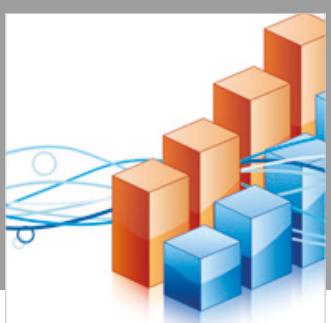

Advances in

Operations Research

\section{-n-m}
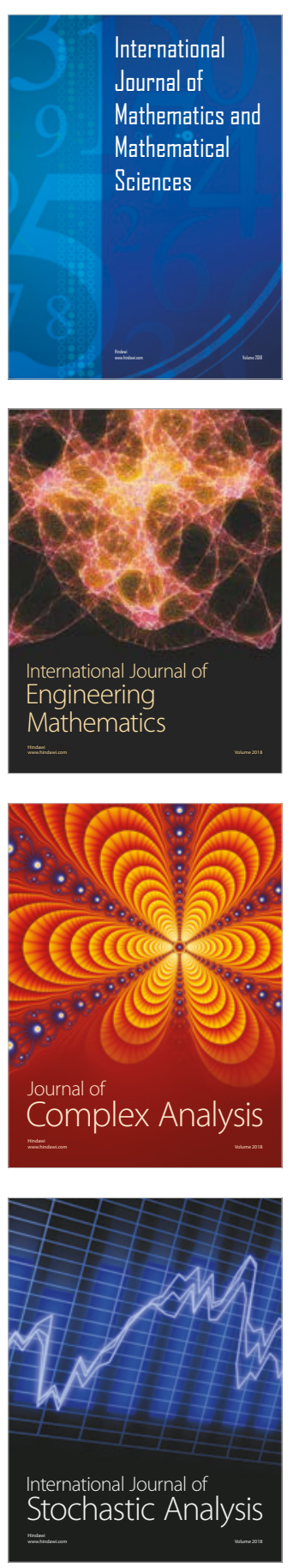
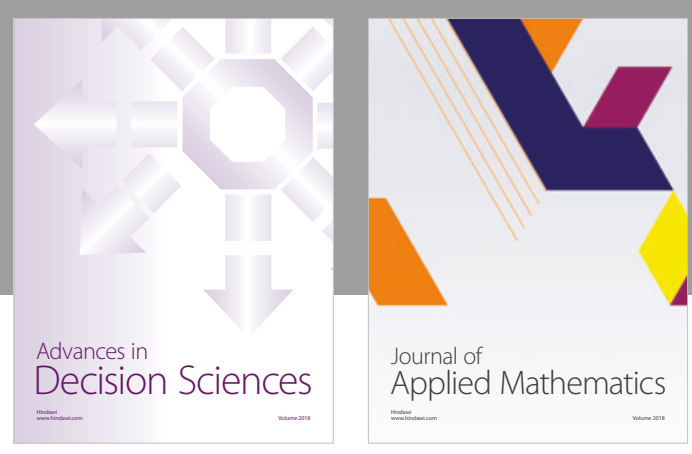

Journal of

Applied Mathematics
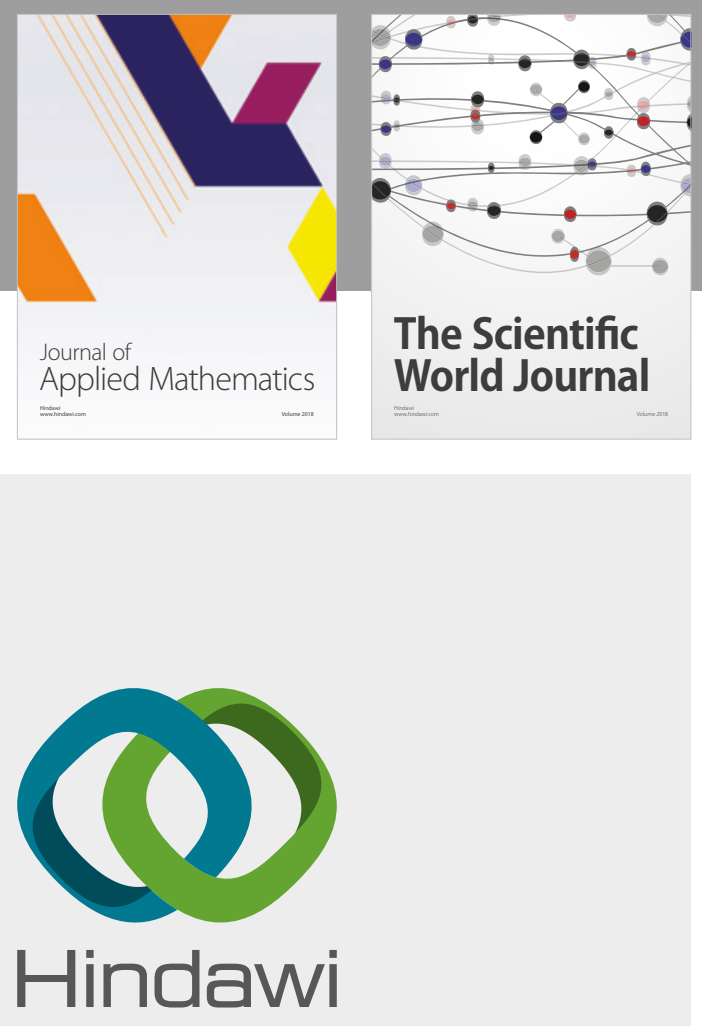

Submit your manuscripts at

www.hindawi.com

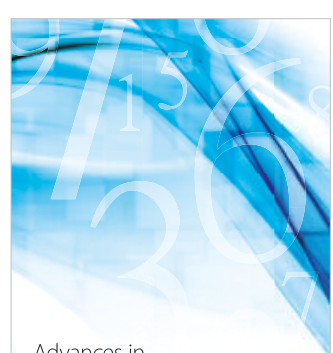

Advances in
Numerical Analysis
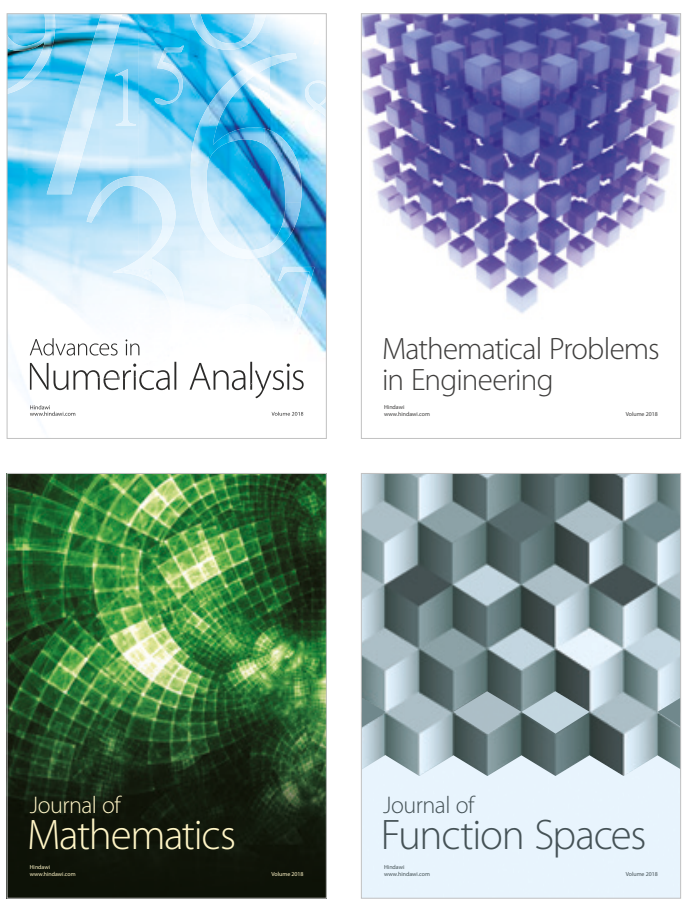

Mathematical Problems in Engineering

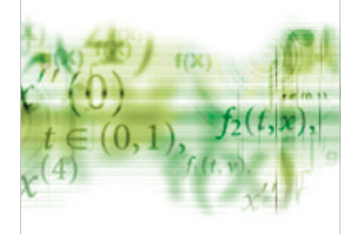

International Journal of

Differential Equations

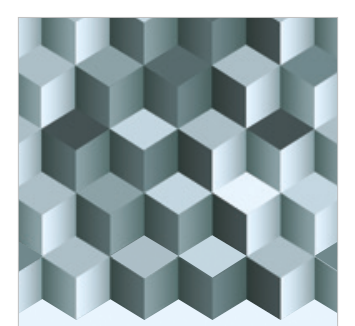

Journal of

Function Spaces

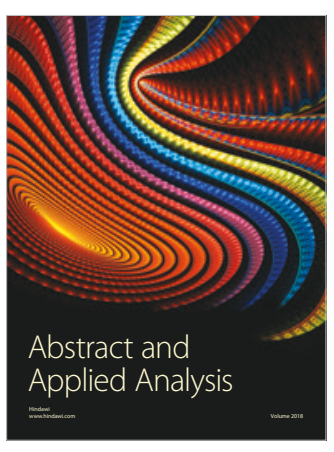

The Scientific

World Journal

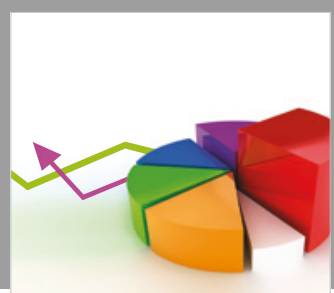

Journal of

Probability and Statistics
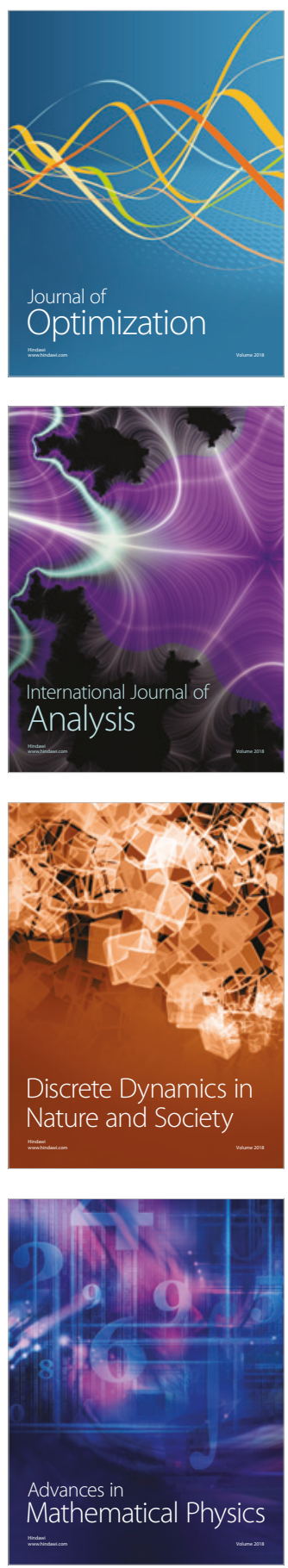